\title{
Use of local neural tube defect registers to interpret national trends
}

\author{
K Hey, M O’Donnell, M Murphy, N Jones, B Botting
}

\begin{abstract}
To conduct a number of studies into the prevalence of neural tube defects (NTD) in the area covered by the Oxford Record Linkage Study (ORLS), multiple sources were used to build a local register of cases occurring in Oxfordshire and West Berkshire between 1968-1990. One source of potential cases - namely, termination and congenital malformation monitoring data available for the locality from the Office of Population Censuses and Surveys (OPCS) data - were kept separate. Comparison of the local cases recorded by OPCS and those known to the register from 1974-1990, using the method of capture-recapture, suggested that national data are only about two thirds complete, but that this underreporting is likely to be reasonably consistent from year to year. OPCS data can therefore be used to study NTD trends if not absolute risks. The local register seemed, by the same yardstick, to be very complete and is being used in a variety of studies of the occurrence of NTD. Survival to one year in this area, over the period 1968-1990, has only improved in the recent past, if at all. Most NTD pregnancies now end in termination rather than birth, and there has been a true decline in the occurrence of NTDs, and likewise the different subtypes. (Arch Dis Child 1994; 71: F198-F202)
\end{abstract}

The Oxford record linkage study (ORLS) is assembled from computed abstracts of hospital inpatient and day case records, together with birth registrations of all babies born in the area and death certificates for them and all current residents of the area. Data relating to the same individual can be linked together and the resulting records analysed to give unduplicated counts and time-sequenced information about people who have been admitted to hospital. ${ }^{1}$

The maternity subset of the ORLS at present includes all hospital maternity events in Oxfordshire and West Berkshire from 1970 to 1986 , with 1965 to 1969 and post 1986 records expected to be added during 1994. From 1973 onwards maternity events preceded by a history of infertility and its treatment were noted, prompting us to conduct a historical prospective study of the relation between successful infertility treatment and subsequent outcome of the pregnancy as neural tube defect (NTD). ${ }^{2}$ For this we needed a register of babies with NTD, born or terminated in Oxfordshire and West Berkshire. We therefore supplemented the cases already known to the ORLS with data about others (largely terminations) from a variety of sources, and extended the period within which cases were defined. As a result, we identified nearly 900 cases across the two districts between 1968 and 1990, complementing and overlapping cases defined for the area in two earlier studies. ${ }^{34}$

Although the ORLS core data were supplemented by a number of information sources (notably the screening programmes), the major independent source of data was the Office of Population Censuses and Surveys (OPCS). Voluntary notification of birth defects to the national congenital malformation monitoring system, and statutory notifications of terminations and the grounds on which they were performed, provide an independent count of the prevalence at birth or at termination of NTD in local areas.

By distinguishing throughout, as part of the study design, between cases identified locally to build the register and national OPCS data available for the locality, we were able to apply the statistical technique of 'capture-recapture' to measure the relative completeness of each of these data bases. ${ }^{5-9}$ Capture-recapture methods derive their name from techniques used to estimate the size of wildlife populations. A sample of the population (of unknown size) is captured, marked, and released to mingle freely again. A second sample of the same population is then taken. Knowing the sizes of the two samples and the number of marked animals from the first sample found also among the second sample, the size of the unknown population can be estimated. Applied to two databases which separately estimate a true number of cases independently of each other, the parallels are the count of cases known to each register and the overlap between them. The central assumption of this technique is independence - that each case is equally likely to be enumerated in either database. This can reasonably be assumed here as birth notifications via the district community health offices and by the physicians undertaking the abortion are the usual source of the OPCS data and our local register was built entirely without recourse to those sources. We can therefore estimate more completely the true prevalence at birth or at termination of NTD locally and hence nationally throughout the study period, by considering the local areas as a typical sample of England and Wales.

We report here the results of building a register of NTDs. The capacity to monitor the trends locally and nationally may be of some value in estimating the effects of folate supplementation in the future. More detailed 


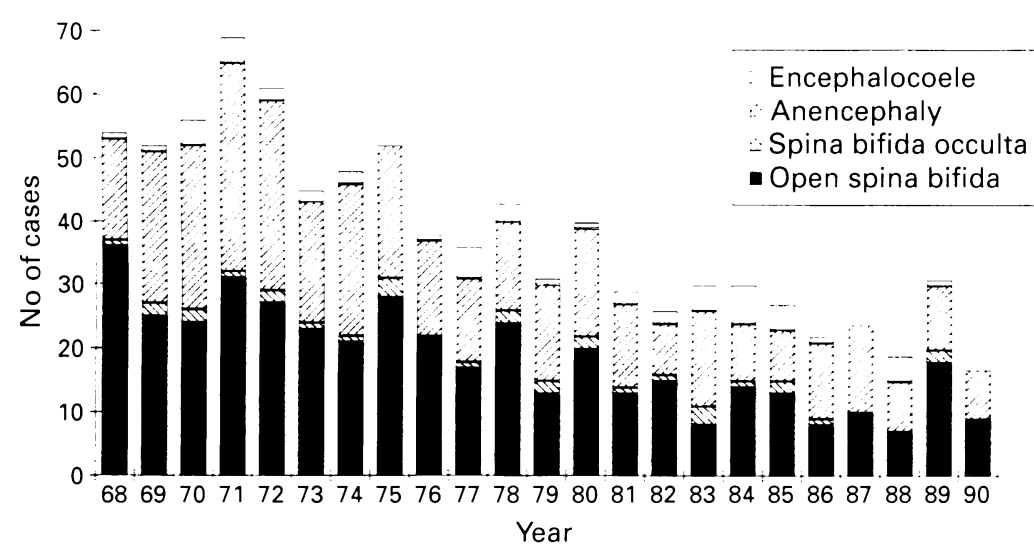

Figure 1 Distribution of types of neural tube defect in Oxfordshire and West Berkshire 1968-90.

consideration of local time trends, seaonality, and descriptive epidemiology will be published elsewhere (Seagroatt V, unpublished observations).

\section{Methods}

We examined all maternity records on the ORLS (undelivered, delivered, and admitted during the puerperal period) with a diagnosis of NTD, whether present in the delivered baby, detected by screening and terminated, or reported in a previous pregnancy. More detailed six digit codes for mother and baby, developed by Jean Golding from four digit codes in the International Classification of Diseases (ICD) revisions 8 and 9, were used at the ORLS from 1973 onwards, and provide even greater specificity.

We also searched the ORLS general hospital file (general hospital admissions, births, and deaths) for any therapeutic abortions performed in a gynaecology ward for NTD, and for any death or stillbirth registrations which reported NTD as a main or underlying cause of death. Until recently ORLS received from OPCS copies of all stillbirth and death certificates for babies born in the two districts and semi-multicause coding of death certificates is undertaken routinely locally. As well as computerised records, we also had available a card index system of congenital abnormalities noted on any ORLS record between 1963 and 1977, arranged by diagnosis.

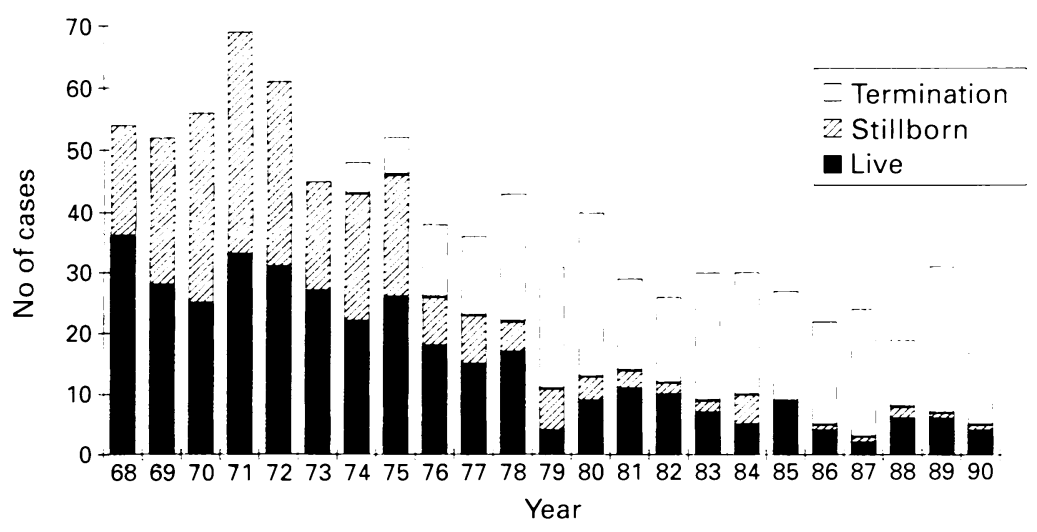

Figure 2 Neural tube defect pregnancies by outcome in Oxfordshire and West Berkshire $1968-90$.
The other major local source of cases was the records of the $\alpha$ fetoprotein (AFP) screening programmes, from 1976 in Oxfordshire and from 1977 in West Berkshire, which recorded the detection and outcome of most NTD pregnancies screened over the period. ${ }^{1011}$ These records most importantly contributed details of terminations which, although usually present on the ORLS general hospital file as operations, tend not to be recorded as NTD related, and hence were unidentifiable.

Although most of the local register was assembled from these two sources, we also acquired cases from perinatal pathology reports and paediatric surgery records. We also checked home birth, delivery suite, operation and abortion registers, and the records of the regional genetics unit including an extant review of about 150 NTD pathology reports. ${ }^{12}$

The third major source of information was OPCS. It is a statutory requirement that all abortions be notified to the chief medical officers of England and Wales who pass them to OPCS for analysis, and one of us who works at OPCS (MM) was able to check details of anonymised data on NTD terminations for the two districts from 1968 to 1990 . We were also able to check similar data from 1974 to 1990 on babies born with NTD in the two districts, notified to the voluntary OPCS national congenital malformation monitoring system. Matching both these datasets against the local register and ORLS general hospital files, using the woman's date of birth, date of operation or delivery, malformation type and geographical code, established a few new cases and the extent of overlap of the local register and nationally derived local dataset.

The woman's general hospital and maternity notes were examined for every potential case to confirm the NTD diagnosis, and to extract details of the index pregnancy, the mother's gynaecological history and her previous and subsequent obstetric history. Post mortem examination reports were available for $60 \%$ of the non-surviving cases, to verify or to expand diagnoses; similarly, several sets of case notes for the surviving children were checked to confirm diagnosis and outcome.

\section{Results}

The NTD register comprises 880 cases identified in Oxfordshire and West Berkshire among residents and non-residents between 1968 and 1990.

Where more than one NTD was present in an individual, anencephaly was counted as the definitive condition in combination with other neural tube defects, while spina bifida aperta or cystica was treated as definitive in combination with any defect other than anencephaly. The register thus comprises 372 anencephalics, 426 cases of spina bifida aperta or cystica, 51 encephalocoeles and 31 cases of spina bifida occulta. Three hundred and fifty seven cases had one or more additional defects, such as hydrocephalus (153 instances), talipes (109 instances). Figure 1 shows the distribution over time for types of NTD in the study period 
Table 1 Survival of NTD livebirths to one year, 1968-1990, in Oxfordshire/West Berkshire

\begin{tabular}{|c|c|c|c|c|c|c|}
\hline \multirow[b]{2}{*}{ Year } & \multicolumn{3}{|c|}{$\begin{array}{l}\text { Spina bifida aperta } \\
\text { (no anencephalus) }\end{array}$} & \multicolumn{3}{|c|}{$\begin{array}{l}\text { Encephalocoele (no anencephalus } \\
\text { or spina bifida aperta) }\end{array}$} \\
\hline & Total & $\begin{array}{l}\text { Dead under } \\
1 \text { year }\end{array}$ & $\begin{array}{l}\text { Dead } \\
\%\end{array}$ & Total & $\begin{array}{l}\text { Dead under } \\
1 \text { year }\end{array}$ & $\begin{array}{l}\text { Dead } \\
\%\end{array}$ \\
\hline \multicolumn{7}{|l|}{ Before screening } \\
\hline $1968-71$ & 100 & 54 & 54 & 7 & 3 & 43 \\
\hline $\begin{array}{l}1972-76 \\
\text { After screening }\end{array}$ & 103 & 60 & 58 & 5 & 2 & 40 \\
\hline $1977-81$ & 36 & 23 & 64 & 10 & 6 & 60 \\
\hline $\begin{array}{l}1982-90 \\
\text { All }\end{array}$ & 31 & 9 & 29 & 12 & 2 & 17 \\
\hline $\begin{array}{l}1968-90 \\
\chi^{2} \text { trend }(1 \mathrm{df}) \\
\chi^{2} \text { hetero }(3 \mathrm{df})\end{array}$ & 270 & $\begin{array}{l}146 \\
2 \cdot 2(p=0 \cdot 14) \\
9.9(p=0.02)\end{array}$ & 54 & 34 & $\begin{array}{l}13 \\
1 \cdot 0(p=0 \cdot 3) \\
4 \cdot 4(p=0 \cdot 2)\end{array}$ & 38 \\
\hline
\end{tabular}

(multiple defects counted as single cases). There were no significant differences in the rates of decline of these groupings suggesting no aetiological heterogeneity between them, ${ }^{13}$ though what has caused the overall decline is not known. ${ }^{14} 15$

There were 355 live births, 237 stillbirths, 277 terminations, and 11 late miscarriages. Although the total prevalence rate (at birth or at termination) of NTD has declined over the study period, as has been found elsewhere (Seagroatt V, unpublished observation), ${ }^{1415}$ the proportion of live and stillbirths has fallen dramatically, matched by a corresponding increase in terminations following the introduction of the screening programme. ${ }^{16}$ Figure 2 shows the changing pattern of outcomes over time during the study period.

Table 1 indicates the proportion surviving their first year of those children born alive with

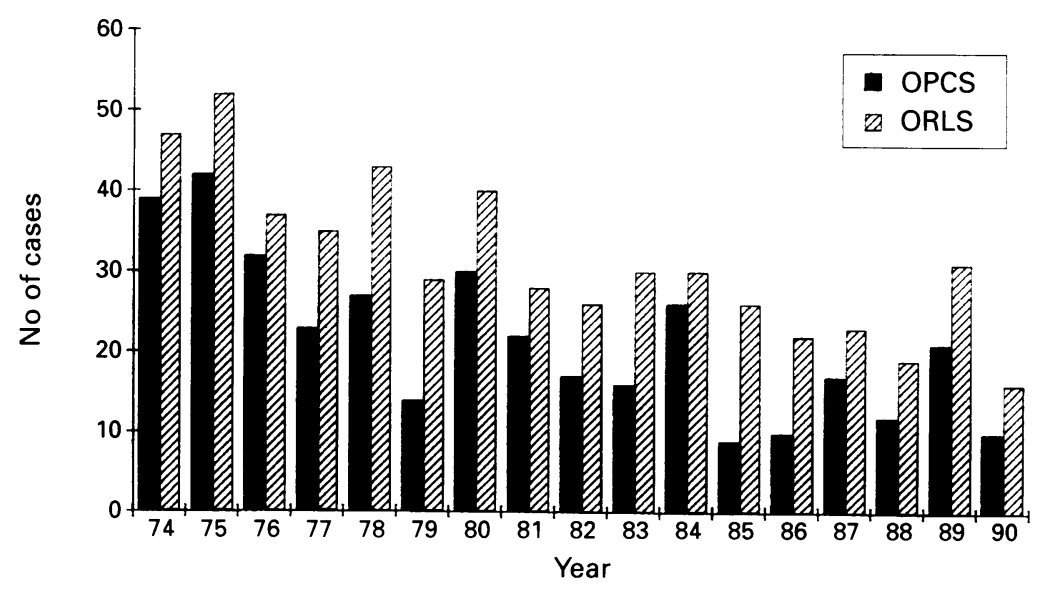

Figure 3 Comparison of number of cases of NTDs recorded by OPCS and ORLS $1974-90$

Table 2 Completeness of ascertainment of NTDs occurring in Oxfordshire and West Berkshire by ORLS register and OPCS data for locality 1974-90

\begin{tabular}{|c|c|c|c|c|c|}
\hline \multirow{4}{*}{$\begin{array}{l}\text { NTDs known } \\
\text { to ORLS }\end{array}$} & \multirow{4}{*}{$\begin{array}{l}\text { Yes } \\
\text { No }\end{array}$} & \multicolumn{2}{|c|}{$\begin{array}{l}\text { NTDs known } \\
\text { to OPCS }\end{array}$} & \multirow{4}{*}{\multicolumn{2}{|c|}{$\begin{array}{l}\text { True number of } \\
\text { NTDs }(T)=a+b+c+d\end{array}$}} \\
\hline & & \multirow{3}{*}{$\begin{array}{l}Y e s \\
a=352 \\
c=15\end{array}$} & No & & \\
\hline & & & $\mathrm{b}=182$ & & \\
\hline & & & $\begin{aligned} d & =\frac{b c}{a+1)} \\
& =8\end{aligned}$ & & \\
\hline \multicolumn{3}{|l|}{ Known to ORLS } & & & $\%$ of $T$ \\
\hline \multicolumn{3}{|l|}{$\begin{array}{l}\text { Known to OPCS } \\
\text { Known to both }\end{array}$} & $=$ & 534 & 96 \\
\hline \multicolumn{3}{|c|}{ Known to both } & $=$ & 352 & $\begin{array}{l}00 \\
63\end{array}$ \\
\hline \multirow{2}{*}{\multicolumn{3}{|c|}{$\begin{array}{l}\text { Known to both ORLS/OPCS combined } \\
\text { True number }(T) \text {, with } 95 \% \text { confidence interval }\end{array}$}} & $=$ & 549 & 99 \\
\hline & & & $=$ & $557(550-564)$ & $100 \%$ \\
\hline
\end{tabular}

NTDs other than anencephaly. There is no obvious trend in improved survival across the period, though some evidence from the heterogeneity of the proportions surviving that there has been an improvement in the 1980s.

Figure 3 compares the total number of cases in the local register with the number of local cases notified as births or abortions to OPCS in each year 1974-90. There is a greater number known to the register than to OPCS, suggesting that national data are incomplete, but this is relatively consistent from year to year.

Table 2 shows the total number of cases 1974-90 known only to our local register, the number known only to OPCS, and the overlap group ascertained by both the register and OPCS. Assuming the independence of the probability of ascertainment of each NTD case by OPCS or the local register, capture-recapture calculation shows that the local register ascertained perhaps $96 \%$ and OPCS perhaps $66 \%$ of the true number of local cases. Enhancing the local register cases with data from OPCS suggests the local register is then $99 \%$ complete, and that the true number of NTDs over the period (with $95 \%$ confidence interval) is 557 (550-564).

Figure 4 shows the most precise estimate of total NTD prevalence rates from 1974-92 in England and Wales that may be determined at the moment using the OPCS abortion and malformation surveillance notifications coded to the eighth and ninth revisions of the ICD. If the underascertainment of NTD cases by OPCS in the Oxford and West Berkshire areas was typical then the true NTD prevalence at birth or termination nationally is about half as much again. ${ }^{16} 17$

Judgment about the contribution made by the different data sources to our register is to some extent arbitrary, because to the extent that there was substantial overlap between some of them, a primary contribution was made by the sources accessed first. The Hospital Activity Analysis (HAA) data of the ORLS was our starting point and contributed 618 cases between 1968 and 1986 . The records of the screening programmes contributed a further 224 cases (as well as 375 overlap cases with ORLS) between 1973-1990. The card indexes yielded seven new cases, and there were 13 stillbirth and death index cases, five paediatric surgery record cases, and five delivery register cases. Perinatal pathology reports contributed two new cases, and two unreported instances of recurrence were found in the case notes.

The OPCS data for the locality identified four notified terminations, previously unknown to the local register, but otherwise largely confirmed cases already known to it. The local register had 55 NTD live or stillbirths apparently unreported to the OPCS malformation surveillance system.

\section{Discussion}

There are comparatively few opportunities with local or national routine information systems to check the quality of the recorded 


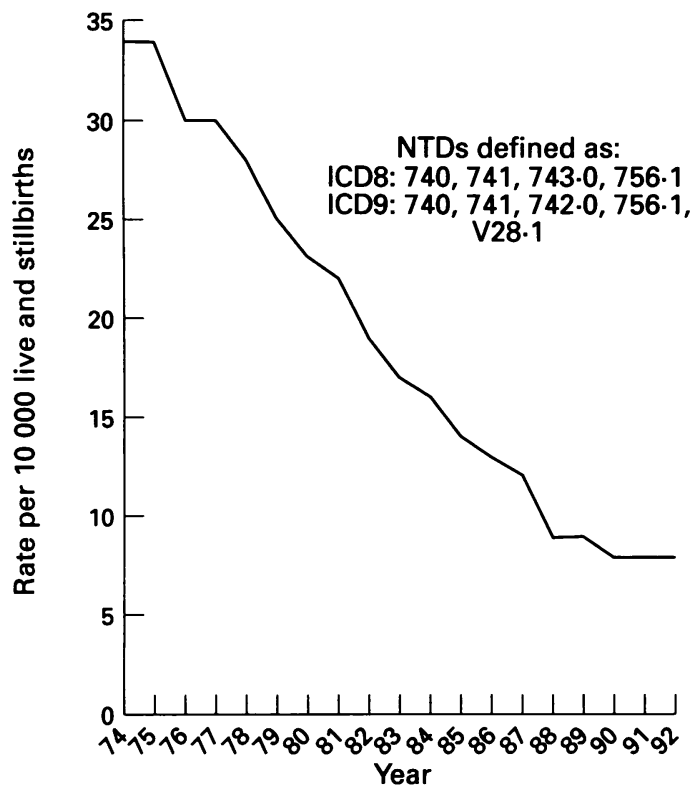

Figure 4 NTD prevalence (at birth/termination) in England and Wales derived from OPCS abortions and birth notifications 1974-92.

data. Building a multisource register provided the opportunity to check both the local (ORIS) computer files and the national (OPCS) datasets with respect to completeness and accuracy.

As diagnosis was the basis of the extracted ORIS maternity file, we were interested in the accuracy of the diagnostic selection and coding. There were 44 ORLS false positive results (three due to screening error, 12 through revision of diagnosis, and 29 through ORLS clerical or coding error) and a small group of possible cases had to be discarded simply because of lack of specificity in the codes assigned - for example, non-spina-bifida occulta correctly coded to ICD9 756.1.

The 29 ORIS false positive results arose during the process of trained staff extracting data from case notes and coding it before input by key to disk. There were four extraction errors where case notes did not confirm the diagnosis, 12 coding errors, such as looking up 'hydromyelocoele' when the extractor had written 'hydrocele', and 13 coder's or keyer's number inversion - where 765100 (premature baby) was recorded but taken as 756100 (spina bifida occulta).

A more subtle check on data quality was a comparison between the diagnostic code of confirmed cases known to the ORLS maternity file and the detailed diagnosis given in the case notes; in other words the fifth and sixth digit accuracy, beyond the basic ICD9 identification. The ORLS maternity file extraction produced 518 validated cases between 1970 and 1985. Of these, 17 had extraction errors, involving misspellings or imprecise terms. There were also seven coder's errors, usually failing to record that the spina bifida lesion was occulta. There were four incomplete diagnoses due to the design of the pre-1973 ORLS data collection forms, and a further three discrepancies arising from revisions of the clinician's diagnosis after the maternity data had been recorded. In all, this gives a $6 \%$ rate of 'fine-tuned' diagnostic error - that is, the NTD condition was correctly identified but the detailed diagnostic coding could have been better.

This overall picture of diagnostic coding in ORLS is broadly reassuring, given that NTDs are often complicated to classify. On the basis of this study, coding, keying, or clerical error caused the ORLS maternity file to miss about $6 \%$ of cases (29 of 518 validated cases), to ascribe adequate but imperfect codes to another $6 \%$ ( 31 of 518 ), and to include $5 \%$ of false positives (29 of 586 extracted possible NTD cases). A comparison was also made of other variables extracted for the 429 validated cases for whom clinical details were already known to the ORIS, having been added to the database over the previous 20 years. This, too, was reassuring, reaching a high level of exact agreement for a number of variables, such as blood group $99 \%$, sex $98 \%$, delivery date $100 \%$, mother's age $97 \%$, and parity $94 \%$.

Home births presented further difficulties, particularly during the early years of the study when they were relatively numerous. They were not routinely recorded on the ORIS maternity file, and we had to assemble them from a range of sources. Individual midwives kept their own records of home deliveries, but these were not centralised or easily available to us within the two districts. Death and stillbirth indexes, the congenital anomalies card index, and paediatric surgery records yielded a total of 13 cases born at home, but we have no direct indication of the extent to which this may underestimate the true number of home delivered babies with NTDs.

Another area of concern was the identification of NTD terminations, particularly before systematic screening began. Although some terminations appear on the ORLS maternity file with an NTD diagnosis, many appear only on the general hospital admissions file, and usually without enough diagnostic data to identify them. For example, eight abortions known to OPCS, the $\alpha$ fetoprotein screening programme, and confirmed in the case notes, were entirely absent from the ORLS general hospital file, although the women had other general and maternity events on file; a further 18 cases were on the general hospital file, but with no indication of NTD. The methods and quality of recording of NTD abortions on ORLS vary greatly between districts and between hospitals within districts, which implies that this is more a problem of source information than of ORLS coding or linkage. Hence data on terminations obtained from Hospital Episode Statistics (HES) or HAA data may not represent a gold standard. ${ }^{18}$ Nevertheless, in general we found that HAA-type data may be a valuable resource for building a register retrospectively, when supplemented by other sources.

Comparison with the local register suggests that OPCS data about prevalence of NTD at birth or termination has some limitations, in that, if Oxfordshire and West Berkshire are typical, the national rate is underestimated by about one third. ${ }^{1617}$ However, underreporting 
seems to be reasonably consistent year by year, and OPCS national data tell the same story, in terms of trend, as the ORLS register from 1974 to 1990 . The statistical stability of national data must be greater, and we judge that it is likely that OPCS data for England and Wales can be used to monitor trends, if not absolute risks. The reasons for the fall in NTD prevalence at birth or termination remain unclear, as does the stability of rates in other areas, ${ }^{19}$ but we should be able to use the data to monitor the effects of folate supplementation nationally, and locally where registers exist. $^{20}$

In contrast, the completeness of OPCS death registration data may be indispensable for local studies of survival. We checked the vital status of all babies not known to have died including anonymised adoptions and those who moved from the area, at the NHS Central Register in Southport and were able to compute full life table follow up until December 1992. Most deaths occurred in the first year and hence we focused on this period, given the relatively small numbers of liveborn infants with NTD known to our local register. Our hypothesis about survival was that there would be a trend to its improvement over the time period, because of selective termination of the more severe NTDs and because of improvement in management of the condition. We have not adjusted in this study for concomitant factors affecting survival, such as level of spinal cord lesion, presence of hydrocephalus, or other major defects and infection, nor did we assess morbidity or quality of life. However, any improvement in survival in this treatment centre, if present at all, seems only to have occurred in the recent past. Nationally, survival to one year has previously displayed a fluctuating pattern over time, perhaps partly due to changing selection policies for surgical treatment. ${ }^{21-23}$

The structure and scale of the NTD register has allowed us to look at trends over time and the descriptive epidemiology of the condition in this medium prevalence area of England and Wales (Seagroatt V, unpublished data). We are also undertaking a case control investigation into a number of factors associated with the occurrence of NTDs, partly to check the findings of our historical prospective study in relation to previous infertility. For this we are selecting matched controls from the ORIS maternity file and, exactly as for the cases, verifying and expanding the computerised information through reference to the hospital case notes. The register, which could now be updated from year to year (and extended back to 1965), can be used to study a variety of issues because of the possibility for record linkage within ORLS. Investigation of recurrence risk and familial disease associations is planned, and we would be pleased to discuss any further suggestions readers may make. ${ }^{24}$

We are very grateful to the Association for Spina Bifida and Hydrocephalus (ASBAH) for funding the initial phase of the study, and to Oxford University Medical School Medical Research Fund for further support.

Special thanks are due also to Dr Alison Milne in West Berkshire for supplying information from the screening programme there. We als thank the obstetricians and gynaWe the obstetricians and gynaecologists of Oxfordshire and West Berkshire for allowing us
access to their patients' case notes.

The Unit of Health Care Epidemiology is part of the Department of Public Health and Primary Care at the University of Oxford, and is funded by the Department of Health. It also receives support from Oxford Regional Health Authority. We thank Unit members, Liza Brandon, for typing this manuscript, and Valerie Seagroatt, for statistical advice about survival analysis.

The views expressed do not necessarily represent those of OPCS or the Department of Health.

1 Acheson ED. Medical record linkage. Oxford: Oxford University Press, 1967

2 Mills JL, Simpson JL, Rhoads GG, Graubard BI, Hoffman $\mathrm{H}$, Conley $\mathbf{M}$, et al. Risk of neural tube defects in relation to fertility and fertility drug use. Lancet $1990 ; 336: 103-4$.

3 Fedrick J. Anencephalus in the Oxford record linkage study area. Devel Med Child Neurol 1976; 18: 643-56.

4 Hobbs MST. Risk of anencephaly in migrant and nonmigrant women in the Oxford area. $\mathrm{Br} \mathcal{F}$ Prev Soc Med 1969; 23: 174-8.

5 Hook EB, Regal RR. The value of capture-recapture methods even for apparent exhaustive surveys. $A m \mathcal{F}$ Epidemiol 1992; 135: 1060-7.

6 Laporte RE, Tull ES, McCarty D. Monitoring the incidence of myocardial infarctions: applications of capture-mark recapture technology. Int $\mathcal{f}$ Epidemiol 1992; 21: 258-62.

7 Laporte RE. Assessing the human condition: capture-recapture techniques allow accurate counts of those difficult to reach populations. $B M \mathcal{F} 1994 ; 308: 5-6$.

8 McCarty DJ, Tull ES, Moy CS, Kwoh CK, Laporte RE. Ascertainment corrected rates: applications of captureAscertainment corrected rates: applications of capture
recapture methods. Int $\mathcal{F}$ Epidemiol 1993; 22: 559-65.

9 Hook EB, Regal RR. Effect of variation in probability of ascertainment by sources ('variable catchability') upon 'capture-recapture' estimates of prevalence. $\mathrm{Am}$ Epidemiol 1993; 137: 1148-66.

10 Williams RM, Sefton C, Milne A, Holt EM, Whyman AE, Alexander I, et al. Three years experience of routine maternal alphafetoprotein screening in a provincial health district. $₹$ Obstet Gynaecol 1982; 2: 145-8.

11 Woolfson J, Holt EM, Whyman AE, Mabbs DV. Materna serum alphafetoprotein screening in a provincial health serum alphafetoprotein screening in a provin
district. Br f Obstet Gynaecol 1979; 86: 87-90.

12 Holmes-Siedle M, Dennis J, Lindenbaum RH, Galliard A. Long term effects of periconceptual multivitamin supplements, for prevention of neural tube defects: a seven to ten year follow-up. Arch Dis Child 1992; 67: 1436-41.

13 Khoury MJ, Erickson JD, James LM. Etiologic heterogeneity of neural tube defects: clues from epidemiology. Am $\mathcal{F}$ Epidemiol 1982; 115: 538-48.

14 Carstairs V, Cole S. Spina bifida and anencephaly in Scotland. $B M \mathcal{F}$ 1984; 289: 1182-4.

15 Stone DH, Smalls MJ, Rosenberg K, Womersley J. Screening for congenital neural tube defects in a high risk area: an epidemiological perspective. $f$ Epidemiol area: an epidemiological perspect

16 Cuckle HS, Wald NJ. The impact of screening for open neural tube defects in England and Wales. Prenatal Diagnosis 1987; 7: 91-9.

17 Cuckle HS, Wald NJ, Cuckle PM. Prenatal screening and diagnosis of neural tube defect in England and Wales in 1985. Prenatal Diagnosis 1989; 9: 393-400.

18 Walsworth-Bell JP. Under-reporting of medical terminations of pregnancy. Br f Obstet Gynaecol 1992; 99: 931.

19 Chan A, Robertson EF, Haan EA, Keane RJ, Ranieri E Carney A. Prevalence of neural tube defects in South Australia, 1966-91: effectiveness and impact of prenatal Australia, 1966-91: effectiveness and
diagnosis. BMF 1993; 307: 703-6.

20 Wald NJ, Bower C. Folic acid, pernicious anaemia and prevention of neural tube defects. Lancet 1994; 343: 307

21 Bradshaw J, Weale J, Weatherall J. Congenital malformations of the central nervous system. Population Trends 1980; 19: 13-18.

22 Weatherall JAC. A review of some effects of recent medical practices in reducing the number of children born with congenital abnormalities. Health Trends 1982; 14: 85-8.

23 McCarthy GT. Treating children with spina-bifida: an individual programme for each child. $B M F 1991 ; 302$ : 65-6.

24 Janerich DT, Mayne ST, Thompson WD, Stark AD, Fitzgerald EF, Jacobson HI. Familial clustering of neural tube defects and gastric cancer. Int $₹$ Epidemiol 1990; 19: 516-21. 